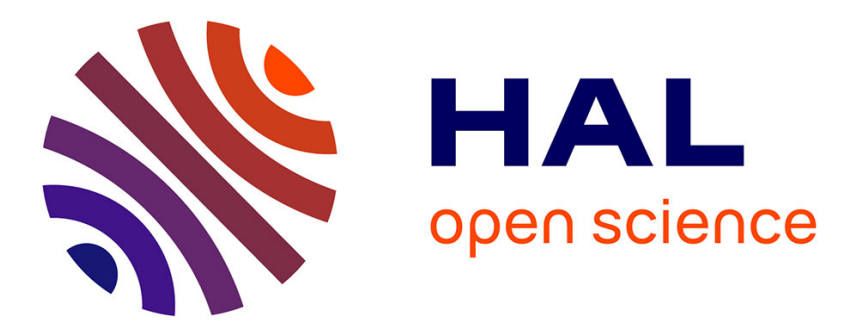

\title{
A new figure of merit for wideband vibration energy harvesters
}

\author{
Wei Qun Liu, Adrien Badel, Fabien Formosa, Yi Peng Wu
}

\section{To cite this version:}

Wei Qun Liu, Adrien Badel, Fabien Formosa, Yi Peng Wu. A new figure of merit for wideband vibration energy harvesters. Smart Materials and Structures, 2015, 24 (12), pp.125012. 10.1088/09641726/24/12/125012. hal-01322274

\section{HAL Id: hal-01322274 \\ https://hal.univ-smb.fr/hal-01322274}

Submitted on 15 Dec 2019

HAL is a multi-disciplinary open access archive for the deposit and dissemination of scientific research documents, whether they are published or not. The documents may come from teaching and research institutions in France or abroad, or from public or private research centers.
L'archive ouverte pluridisciplinaire $\mathbf{H A L}$, est destinée au dépôt et à la diffusion de documents scientifiques de niveau recherche, publiés ou non, émanant des établissements d'enseignement et de recherche français ou étrangers, des laboratoires publics ou privés. 


\title{
A new figure of merit for wideband vibration energy
}

\section{harvesters}

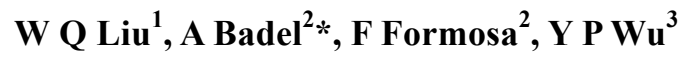 \\ ${ }^{1}$ School of Mechanical Engineering, Southwest Jiaotong University, 610031, Chengdu, China \\ ${ }^{2}$ Laboratoire SYMME, Université Savoie Mont Blanc, 74000, Annecy, France \\ ${ }^{3}$ The Key Laboratory of Education Ministry on Aircraft Structural Mechanics and Control, \\ Nanjing University of Aeronautics and Astronautics, 210016, Nanjing, China \\ *Corresponding author: adrien.badel@univ-smb.fr
}

\begin{abstract}
The performance evaluation method is a very important part in the field of vibration energy harvesting. It provides the ability to compare and rate different VEHs (Vibration Energy Harvesters). Considering the lack of a well-recognized tool, this article proposed a new systematic figure of merit for the appraisement of wideband VEHs. Extensive investigations are first performed for some classic figures for linear VEHs. With the common fundamental information obtained, the proposed figure integrates four essential factors: the revised energy harvester effectiveness, the mechanical quality factor, the normalized bandwidth and the effective mass density. Special considerations are devoted to the properties of wideband VEHs about the operation range and the average power in this domain which are related to the performance target of stable power output. Afterward, this new figure is applied to some literature VEHs and demonstrated to present good evaluations of wideband VEHs. Moreover, it exhibits the ability to point out the improvement information of the concerned VEHs further developments.
\end{abstract}

\section{Introduction}

The rapid development of the technology of microelectronics and MEMS systems during the past decades brings the power requirement of wearable and/or wireless electronics, such as smart sensors, to dozens of micro watts [1]. Meanwhile, the desire for free mobility and for the release from the constraint of wires is growing rapidly [2]. Wireless technologies are facing the best opportunities ever under the drive of the technologies and the demand, especially for the WSNs (Wireless Sensor Networks). Compared with the wired solutions, a WSN allows portability and reduces installation costs. Significant benefits are exhibited for WSNs in many applications, such as structure health monitoring [3], food and agriculture industry [4], environment monitoring [5], human health care [6], and so on. Though, up to date, most WSNs still use batteries as the power sources, leading to a relatively short life span and high cost of maintenance.

As an emerging technology, energy harvesting has received great concern in the past years because of its prospective applications in the power supply of autonomous sensor networks. Among the different potential energy sources from environment and human activities to be captured for WSNs, such as light [7], heat [8], vibration [9], vibration energy harvesting is one of the most promising solutions because of its considerable power density and wide application possibilities [10, 11$]$.

In general, a VEH (Vibration Energy Harvester) is composed of three parts: a mechanical 
oscillator, a transduction element and an interface circuit [12]. For the conventional VEHs, which utilize linear resonant structures [13-15], usually called as linear VEHs, the performance improvements follow two steps: first, increase the maximal available energy; second, enhance the ratio of the usable power output to the maximal available energy. The realization of a high quality factor structure is the only feasible way for the optimization in the first step when the excitation and the inertial mass are fixed [16]. As for the second step, common solutions are to increase the electromechanical coupling level or to develop high performance circuits. One can list SSHI (Synchronized Switching Harvesting on an Inductor) [17-19], SECE (Synchronous Electric Charge Extraction) [20], DSSH (Double Synchronized Switch Harvesting) [21], OSECE (Optimized Synchronous Electric Charge Extraction) [22, 23] for the piezoelectric VEH, SMFE (Synchronous Magnetic Flux Extraction) [24], a forward-feedthrough and feedback DC-DC PWM boost converter [25] for the electromagnetic VEH etc.

Another very important parameter of the VEHs is their volume, which relies on the optimization of the mechanical structures. Dividing the power by the volume, the power density, a significant evaluation factor of the VEH's performance, can be obtained.

A large number of linear VEHs showing great efforts in the optimization about these concerned issues have been proposed. However, it is difficult to directly compare those VEHs because of the different excitations, structures (inertial mass, volume and transduction element) and circuits. Therefore, a unified evaluation method has to be promoted to make fair comparisons between VEHs. A simple way is to compare the harvested power normalized by the mass and the acceleration according to the power of linear VEHs [16]. Roundy et al. suggested a figure of merit named effectiveness, which contains the information about the coupling level, the mechanical quality factor, the density ratio for a baseline material and the circuit efficiency [26]. It is relatively complex and is not straightforward to reflect the VEH's performance.

In order to provide the information about how close the VEH is to its maximal performance, Mitcheson et al. proposed an alternative FoM (Figure of Merit) which defines the energy harvester effectiveness as the usable power divided by the maximal available power [27]. As a further step, Mitcheson et al. [28] developed the $\mathrm{FoM}_{\mathrm{v}}$ (volume Figure of Merit) to reflect the VEH's nearness to the performance limitation with respect to the same volume and excitation. Beeby et al. put forward the NPD (Normalized Power density) [29] as an evaluation tool with wide acceptance because of its simplicity and intuition. These FoMs make it possible to compare different VEHs according to a selected common criterion. Moreover, the information about how to improve the VEHs' performance is also presented to some extent when evaluating the VEHs with the FoMs.

Since the majority of the realistic excitations are variable and broadband, great challenges are raised for conventional linear VEHs, which exhibit a narrow operation band around their resonant frequency. Consequently, it is critical to have a wideband VEH usable for complex application environments. Great achievements have been obtained in recent years [30-33] with multiple solutions, such as resonance tuning VEHs [34, 35], frequency up-conversion VEHs [36, 37], multimodal VEHs $[38,39]$, nonlinear VEHs [40-45] etc. The investigations show that the operation band of these wideband VEHs is significantly enlarged in contrast to the corresponding linear VEHs. However, the bandwidth improvement effects of various wideband VEHs are different and dependent on the used mechanisms and parameters. Thus, the same question exists for the wideband as the linear VEHs that an evaluation criterion is expected. 
A preliminary way is to exploit the same figures of the linear VEHs such as NPD and FoM $\mathrm{v}_{\mathrm{v}}$ for wideband VEHs as in [46, 47]. Nevertheless, these figures are dedicated to linear VEHs with appraisement only accounted for the maximum power output case at the resonant frequency. The bandwidth information is not included. Moreover, the average power over the operation band is more suitable to be considered as the performance evaluation factor instead of the maximum power out. Indeed the target of wideband VEHs is to provide stable power supply for varied excitation cases. Apart from the linear VEH figures, some simple evaluation methods are also proposed in the literatures, for instance, the product of the normalized bandwidth and the power normalized by the excitation [48]. However, in these cases, the appraisement is incomplete. An all-round figure of merit for wideband VEHs is required to take all the critical characteristics into consideration, including the bandwidth, the average power, the volume, the mechanical quality factor of the structure. By doing this, comparisons between different wideband VEHs can be made while indications for the further improvement of the considered VEH are obtained as well. Indeed, it makes sense to develop a universal and comprehensive FoM of the wideband VEHs, which can be used as a performance evaluation and design directive tool. Although a real design optimization is usually constrained by the actual application, the FoM is capable of providing the information about which solution is more appropriate and how to improve the VEH's performance. Moreover, a custom FoM can be built from the universal FoM and according to a specific application.

In this article, a generic model is proposed first as the discussion basis. Then some classic figures for linear VEHs are investigated in detail to collect the essential information to obtain a most relevant FoM. With the knowledge of the linear VEH figures, a new figure is developed with special attention focused on the average power and the bandwidth considered as the essential features of the wideband VEHs. The common issues for linear VEHs, such as the volume, the mechanical quality factor and the energy harvester effectiveness, are integrated as well. Detailed discussion, which covers the different angles of this new figure, is presented. Finally, the proposed FoM is applied for some wideband VEHs for demonstration.

\section{Analysis and discussion}

\subsection{Generic model}

In order to facilitate the next-step discussions about the figures of merit for VEHs, a generic model shown in figure 1 is proposed for VEHs. As has been suggested in previous studies [49, 45], the energy extracted from the generator can be viewed as an additional damping (denoted $\mu_{e}$ ) in the dynamic model. The assumption of modeling the transducer by a simple damper is valid in the case of sinusoidal ambient acceleration, choosing the value of $\mu_{e}$ such as the energy dissipated into the damper during one mechanical period corresponds to the harvested energy. To be distinguished from the mechanical damper $\mu_{m}, \mu_{e}$ is named as the electronic damping hereafter. The mechanical damper $\mu_{m}$ in figure 1 is a damper that embodies mechanical and electrical losses (if any). Finally, the electronic part of the harvester can be included in the dynamic model and a single governing equation for the VEH is expressed as:

$$
M \ddot{x}+K_{l} x+\left(\mu_{m}+\mu_{e}\right) \dot{x}+F_{n}(x)=M \gamma(t)
$$

where $M$ is the inertial mass; $\gamma(t)$ is the ambient acceleration; $K_{l}$ is the linear stiffness; $\mu_{m}$ is the mechanical damping coefficient; $\mu_{e}$ is the electronic damping coefficient; $F_{n}(x)$ is the nonlinear 
restoring force (if any).

Assuming that all the energy dissipated by the electronic damping (extracted by the interface circuit) is transferred to the load, the transient power scavenged by the harvester is written as:

$P=\mu_{e} \dot{x}^{2}$

Since the harvested power is related to the interface circuit, the load situation, the excitation level and other factors, the electronic damping coefficient may vary during operation. However, with the load value fixed and the influence of the excitation level negligible, $\mu_{e}$ is assumed to be constant for simplifications in this article.

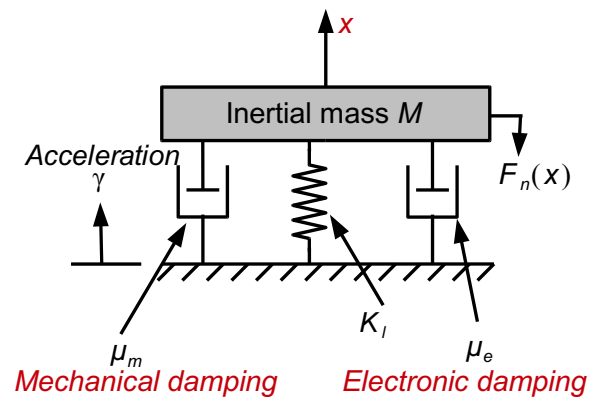

Figure 1. Generic model for VEHs.

For the purpose of obtaining the critical issues about the development of a relevant figure of merit, investigations are firstly devoted to some well-known figures such as PNMA (Power Normalized by the Mass and the Acceleration), NPD (Normalized Power Density) and $\mathrm{FoM}_{\mathrm{v}}$ (volume Figure of Merit) etc.

\subsection{Classic figures of merit for VEHs}

Since the classic figures (PNMA, NPD and $\mathrm{FoM}_{\mathrm{v}}$ ) are first mainly intended for linear harvesters, the assumption of linear case is also applied for the following discussion of these figures. With the nonlinear force $F_{n}(x)$ set to be zero in equation (1), we have the usual linear form:

$$
M \ddot{x}+K_{l} x+\left(\mu_{m}+\mu_{e}\right) \dot{x}=M \gamma(t)
$$

When a harmonic excitation $\gamma(t)=\gamma_{0} \cos \omega t$ is applied, the corresponding velocity solution is also harmonic for a linear harvester. It can be expressed as $\dot{x}=v_{0} \cos (\omega t+\theta)$ in which $\theta$ is the phase difference between the excitation and the velocity. Substituting $\gamma(t)$ and $\dot{x}$ into equation (3), we have:

$$
\left(-M \omega^{2}+K_{l}\right) \omega v_{0} \sin (\omega t+\theta)+\left(\mu_{m}+\mu_{e}\right) v_{0} \cos (\omega t+\theta)=M \gamma_{0} \cos \omega t
$$

The harvester obtains the maximum power around the resonant frequency $\omega=\omega_{0}=\left(M / K_{l}\right)^{1 / 2}$. Then the first term in equation (4) is zero and we can get $\theta=0$, which means that the total damping force and the excitation force are equals and in phase:

$$
\left(\mu_{m}+\mu_{e}\right) \dot{x}=\left(\mu_{m}+\mu_{e}\right) v_{0} \cos \omega_{0} t=M \gamma_{0} \cos \omega_{0} t
$$

Using equations (2) and (5), the average power over a period under the resonant condition is expressed as: 


$$
P_{r e s}=\frac{\omega_{0}}{2 \pi} \int_{0}^{2 \pi / \omega_{0}} \mu_{e} v_{0}^{2} \cos ^{2} \omega_{0} t d t=\frac{1}{2} \mu_{e} \frac{M^{2} \gamma_{0}^{2}}{\left(\mu_{e}+\mu_{m}\right)^{2}}
$$

which represents the peak average output power of the harvester. This value is used in all the classic figures of merit for evaluating the best performance of the concerned linear harvesters. For a VEH with a determined inertial mass and a fixed $\mu_{m}, P_{\text {res }}$ reaches the maximum value:

$$
P_{\max }=\frac{1}{8} \frac{M^{2} \gamma_{0}^{2}}{\mu_{m}}
$$

by letting $\mu_{e}=\mu_{m}$ which means that the electronic damping should exactly match the mechanical damping.

\subsubsection{PNMA.}

The PNMA figure has not been proposed specifically in the literature yet, but has been often used when appraising a harvester, especially for a linear one. The basic idea is to make relatively fair comparisons between different harvesters with the same inertial mass and acceleration. The definition of PNMA is given by equation (8) which is based on the linear harvester's property that the power is proportional to the product of both the mass and the second power of the ambient acceleration.

$$
P N M A=\frac{P}{M \gamma_{0}^{2}}
$$

However, it is worth mentioning that although the PNMA figure is based on the linear harvester assumption, it is still applicable for other kinds of harvesters. Substituting equation (6) into equation (8), we have the PNMA value at the resonant frequency:

$$
P N M A=\frac{1}{2} \frac{M \mu_{e}}{\left(\mu_{m}+\mu_{e}\right)^{2}}
$$

The maximal value is found when the harvested energy equals the energy dissipated by the mechanical damping ( $\mu_{e}=\mu_{m}$ and $P_{\text {res }}$ is maximal). Therefore, to achieve the optimal PNMA value, a proper configuration of the interface circuit, the load and the electromechanical coupling coefficient is required. As explicated by Lefeuvre et al. [50], when the standard circuit (simple full bridge rectifier) is used as the interface circuit, this equilibrium is only obtained for coupling coefficients higher than a specific value and for an optimal load. If the high coupling requirement is not satisfied, nonlinear synchronous switching techniques (SSHI, SECE, OSECE etc [17-23]) are required to increase the electronic damping. Once this equilibrium $\mu_{e}=\mu_{m}$ is reached, equation (9) can be simplified to:

$$
P N M A=\frac{M}{8 \mu_{m}}=\frac{1}{16 \xi_{m} \omega_{0}}
$$

where $\xi_{m}=M /\left(2 \mu_{m} \omega_{0}\right)$ is the mechanical damping ratio which is usually constant for a determined structure. From equation (10), it is seen that better appraisement of the harvester using the PNMA figure is obtained with a higher mechanical quality factor $Q_{m} \approx 1 /\left(2 \xi_{m}\right)$ and a lower resonant frequency $\omega_{0}$.

From the analysis above, it can be found that the PNMA figure has a two-fold meaning: first, for 
the electronic part, the interface circuit should be capable of making the harvested electric energy match with the mechanical dissipation as close as possible; second, for the structural part, high quality factor and low resonant frequency are preferred (for a constant acceleration level). However, the preference to low-frequency structures makes the fairness of the PNMA figure doubted. Usually, a good evaluation tool should have low dependence on the resonant frequency, which is often determined by the application environment.

As seen in the definition, PNMA uses the mass rather than the volume as the normalization parameter. As a result, it lacks the ability of reflecting the compactness of the structure, which is an especially important criterion for the VEHs. An undesirable case is that the inertial mass $M$ might only occupy a small ratio of the total volume of a bulk harvester with a high PNMA value. Especially for the harvesters with a low resonant frequency favored by the PNMA figure, the requirement of large stroke will drastically increase the total occupied volume. Therefore, PNMA is effective for the evaluations of the mechanical quality factor and the matching degree of the interface circuit for linear harvesters with similar structural architecture, yet a more reasonable figure is required to take the volume into account in order to make comparisons between harvesters exhibiting different types of structure. Moreover, the fact that only the power output at a single frequency is considered in the figure makes it not suitable for the evaluations of wideband VEHs.

\subsubsection{NPD.}

As a most widely used figure of merit for VEHs, NPD is firstly proposed by Beeby et al. [29]. It is defined as:

$$
N P D=\frac{P}{V \gamma_{0}^{2}}
$$

which implies the available power for the unit volume and the base value of the second power of the acceleration.

Compared with the PNMA figure, NPD uses the volume instead of the mass for the calculation. To clarify the relation between the two figures, NPD can be rewritten as:

$$
N P D=\frac{P}{M \gamma_{0}^{2}} \times \frac{M}{V}=P N M A \times \rho_{e f f}
$$

It is seen that NPD can be actually expressed by PNMA multiplied by the effective mass density $\rho_{\text {eff }}$. Taking the volume utilization efficiency reflected by $\rho_{\text {eff }}$ into consideration makes NPD provide a more comprehensive evaluation of the VEHs. It is easy to understand that there are two ways to increase $\rho_{\text {eff. }}$ One is to use high-density materials for the inertial mass. The other is to improve the occupation ratio of the inertial mass in the total volume, which forwards the challenge related to the structural optimizations.

When trying to achieve a high NPD, it is interesting to note that high quality factor and low resonant frequency will increase the PNMA value, but decrease the effective mass density in the meantime because of the enlarged motion space of the inertial mass. The competition of these two parameters implies the existence of a possible optimal configuration for $\xi_{m}$ and $\omega_{0}$ to obtain a maximum value of NPD. 


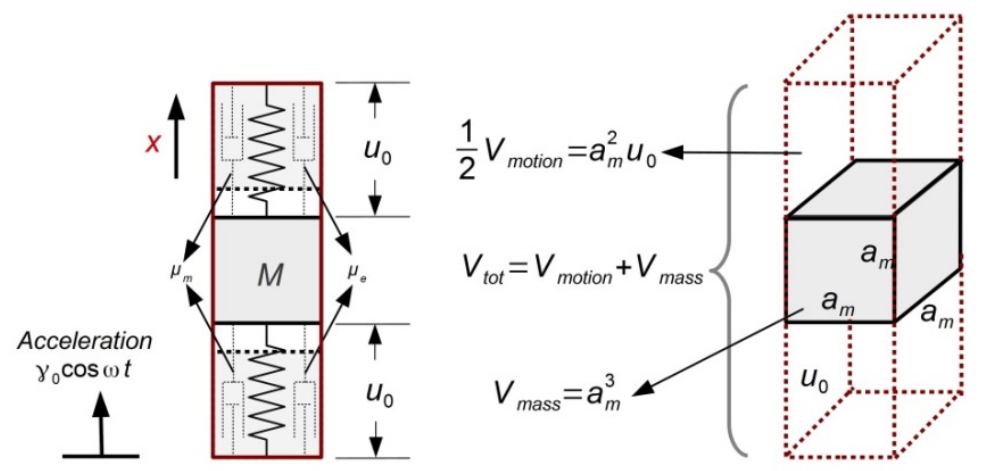

Figure 2. An ideal VEH with the volume only composed of the inertial mass and the motion space.

Case I: The inertial mass is fixed while the volume is variable

Considering the harvester shown in figure 2, the inertial mass is assumed to be cubic $\left(a_{m}{ }^{3}\right)$ while the volumes of other structural parts like springs, transduction elements etc. are negligible for the ideal optimization case. Then the VEH's total volume is approximated by the inertial mass volume $V_{\text {mass }}$ plus the motion space $V_{\text {motion }}$.

Using equation (5) and the assumption of the maximum power with $\mu_{e}=\mu_{m}$ at the resonant frequency, we have the displacement amplitude:

$$
u_{0}=\frac{M \gamma_{0}}{2 \mu_{m} \omega_{0}}=\frac{\gamma_{0}}{4 \xi_{m} \omega_{0}^{2}}
$$

Then the total volume is:

$$
V_{\text {tot }}=V_{\text {mass }}+V_{\text {motion }}=a_{m}^{3}+2 u_{0} a_{m}^{2}
$$

Substituting equations (10), (13) and (14) into equation (12):

$$
N P D=\frac{1}{16 \xi_{m} \omega_{0}} \frac{M}{a_{m}{ }^{3}+\frac{\gamma_{0}}{2 \xi_{m} \omega_{0}^{2}} a_{m}{ }^{2}}=\frac{1}{16 \xi_{m} \omega_{0}+\frac{8 \gamma_{0}}{a_{m} \omega_{0}}} \frac{M}{V_{\text {mass }}}=\frac{\rho_{\text {mass }}}{16 \xi_{m} \omega_{0}+\frac{8 \gamma_{0}}{a_{m} \omega_{0}}}
$$

where $\rho_{\text {mass }}$ is the density of the inertial mass. Obviously in this case, low $\xi_{m}$ is still preferred to obtain high NPD harvesters as PNMA. Meanwhile, there is an optimal $\omega_{0}$ for a determined $\xi_{m}$.

Case II: The volume is fixed while the inertial mass is variable

Considering the harvester in figure 2 as before, the variable inertial mass indicates that $a_{m}$ is to be determined. Then using equations (13) and (14), we can express $\xi_{m}$ with am as follows:

$$
\xi_{m}=\frac{\gamma_{0} a_{m}^{2}}{2 \omega_{0}^{2}\left(V_{t o t}-a_{m}^{3}\right)}
$$

Then equation (15) can be rewritten as:

$$
N P D=\frac{1}{\frac{a_{m}^{2}}{\left(V_{t o t}-a_{m}{ }^{3}\right)}+\frac{1}{a_{m}}} \frac{\omega_{0} \rho_{\text {mass }}}{8 \gamma_{0}}
$$

The maximum value of NPD is obtained for $a_{m}{ }^{3}=V_{\text {tot }} / 2$. It can be inferred that there is an optimal 
configuration of the inertial mass volume and the mechanical damping ratio for the volume preset case. It is similar to the results for FoM $\mathrm{v}_{\mathrm{v}}$ that is presented in the next section. It is interesting to see that the NPD value linearly increases with $\omega_{0}$. The reason is that the work done in each cycle is constant in this case while high $\omega_{0}$ means more cycles during the same time period.

By including the volume into the evaluation, the NPD figure presents two important improvements. First, the structural optimization to promote the compactness of the harvester is advanced. Second, in the mass preset case, the preference to low resonant frequencies is somehow fixed, yet it still shows dependence on $\omega_{0}$ as shown in equation (15); in the volume preset case, an optimal compromise of the mass occupied volume and the mechanical damping is found to achieve the best NPD. However, in case II, NPD shows a proportional relationship to $\omega_{0}$. In general, NPD provides a more reasonable and overall evaluation of the harvesters than PNMA does. However, it is still not suitable for the evaluations of wideband energy harvesters since no bandwidth information is embodied in the definition.

2.2.3 $\mathrm{FoM}_{v}$. In order to get rid of the influence of $\omega_{0}$ and provide a more honest evaluation of the VEHs, a feasible way is to compare the concerned VEH's performance with its maximum possible power output:

$$
E_{H}=\frac{\text { Useful Power Output }}{\text { Maximum Possible Power }}=\frac{\frac{1}{2} \frac{\mu_{e} M^{2} \gamma_{0}^{2}}{\left(\mu_{e}+\mu_{m}\right)^{2}}}{\frac{M^{2} \gamma_{0}^{2}}{8 \mu_{m}}}=\frac{4 \mu_{e} \mu_{m}}{\left(\mu_{e}+\mu_{m}\right)^{2}}
$$

which is labeled as energy harvester effectiveness by Mitcheson et al [27]. It reflects whether the harvester's extracted energy is at the optimal value and approaches the ideal performance. Once the matching condition $\mu_{e}=\mu_{m}$ is satisfied, $E_{H}$ obtains the limitation value of $100 \%$. However, the energy harvester effectiveness is short of distinguishing VEHs with different structural parameters and geometries.

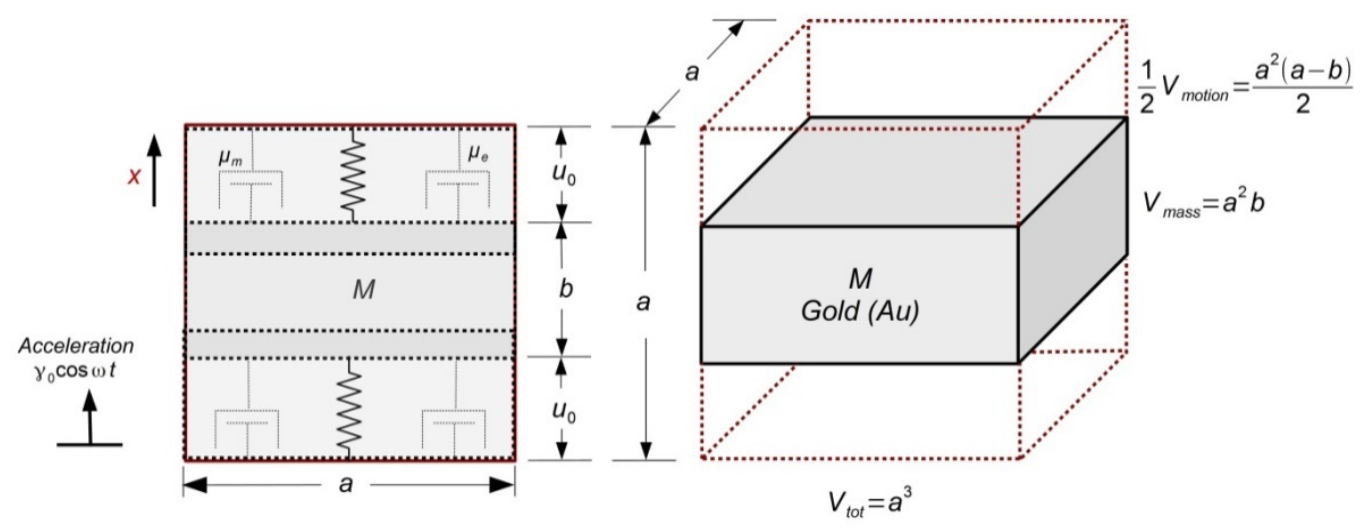

Figure 3. The reference VEH with a cubic volume and an inertial mass of gold ( $\mathrm{Au}$ ). The maximum power is obtained when the mass occupies half of the total volume $(b=a / 2)$.

By further developing this idea, Mitcheson et al. [28] suggested an alternative solution of comparing the considered VEH with a reference harvester. This reference VEH is assumed to have the same volume as the considered VEH with a cubic shape shown in figure 3. The ideal case is still assumed 
that all other parts except the inertial mass retain a negligible ratio of the total volume. To provide a high-level benchmark, the reference device has an inertial mass of gold, which is among the materials of the highest density $\rho_{a u}$. As the performance of the reference generator changes with the ratio variations of the mass's volume to the motion space in the given volume, the best performance of the reference harvester with the optimal volume configuration is to be determined. As shown in figure 3, the given total volume is $V_{t o t}=a^{3}$ (the same as the considered VEH) while the mass volume is $V_{\text {mass }}=a^{2} b$. From the remaining motion space, the displacement amplitude at the resonant frequency is determined as $u_{0}=(a-b) / 2$. Using equation (13), we can find out the corresponding mechanical damping coefficient:

$\mu_{m}=\frac{M \gamma_{0}}{2 u_{0} \omega_{0}}$

It means that for a given volume, there exists an optimal damping coefficient to maximize the power density of the linear VEH subjected to harmonic ambient acceleration. It is worth mentioning that for NPD, this deduction is also applicable if the volume requirement is predetermined while the inertial mass is variable.

Then the peak power output of the reference harvester at the resonant frequency with $\mu_{e}=\mu_{m}$ is calculated:

$$
P_{\text {res-ref }}=\frac{M^{2} \gamma_{0}^{2}}{8 \mu_{m}}=\frac{M \gamma_{0} u_{0} \omega_{0}}{4}=\frac{\omega_{0} \gamma_{0} \rho_{a u} a^{2} b(a-b)}{4}
$$

Obviously, the power apex is found for $b=a / 2$. It means that the optimal performance is obtained with half the total volume occupied by the mass. Then we have the optimal power of the reference generator:

$P_{r e f}=\frac{\omega_{0} \gamma_{0} \rho_{a u} a^{4}}{16}=\frac{\omega_{0} \gamma_{0} \rho_{a u} V_{\text {tot }}^{\frac{4}{3}}}{16}$

Replacing the maximum possible power in equation (18) with $P_{r e f}$, we have the $\mathrm{FoM}_{\mathrm{v}}$ as promoted by Mitcheson [28]:

$$
F o M_{v}=\frac{\text { Useful Power Output }}{P_{\text {ref }}}
$$

$\mathrm{FoM}_{\mathrm{v}}$ is a systematic figure, which has been widely used [37,46]. It is meant to estimate how close the performance of the VEH under evaluation is to the benchmark, which is obtained from the performance limitation of the reference cubic harvester with an identical volume. As the VEH's performance is related to many factors, including the structural architecture, the electromechanical coupling coefficient, the interface circuit, the load and so on, overall considerations have to be taken into account to achieve a high $\mathrm{FoM}_{\mathrm{v}}$. Great efforts are required on structural optimizations about the compactness and the coupling coefficient as well as the realization of the matching condition between the electronic and mechanical damping.

Since all the requirements are integrated as a whole unit in the $\mathrm{FoM}_{v}$, the VEH's evaluation information is not well decoupled. Consequently, it is difficult to know which part of the design imposes the largest influence on the final performance evaluation when calculating the FoM $_{\mathrm{v}}$. The guiding on the improvement of the design is missed to some extent. Meanwhile, the reference 
generator uses gold as the mass material to give a high-level benchmark, it is however not so realistic because of the issues of cost or fabrication or others. It leads to the unfair fact that a VEH with a very good design but normal materials will probably have a lower $\mathrm{FoM}_{\mathrm{v}}$ value than a VEH with a bad design but with materials of high density.

In order to capture further the operation bandwidth information, a derivative figure was also proposed by Mitcheson et al. [28]:

$$
F O M_{B W}=F O M_{v} \times \frac{\delta \omega_{1 d B}}{\omega}
$$

which was named as the bandwidth FoM. The bandwidth is chosen to be the frequency range within which the power is more than the maximum power value minus $1 \mathrm{~dB}$. Nevertheless, FoM $\mathrm{M}_{\mathrm{BW}}$ is still focused on the maximum power performance of the VEH and mainly oriented for linear VEHs.

\section{3 remarks}

From the above analyses about the classic figures based on linear VEHs, some critical indications to develop an effective FoM can be concluded. In general, a good figure is supposed to provide the following evaluations of the concerned VEH:

- $\quad$ Energy harvester effectiveness $E_{H}$.

$E_{H}$ represents the VEH's ability to reach the maximum available power. As seen in equations (7), (10) and (16), the optimal peak power at the resonant frequency is obtained for the matching condition $\mu_{e}=\mu_{m}$, corresponding to a value of $100 \%$ for the energy harvester effectiveness. To achieve this optimal equilibrium, a proper configuration of the interface circuit, the electrical load and appropriate electromechanical coupling coefficient are required.

- Volume $V_{\text {tot }}$.

As mentioned in the former discussions, the volume is a very important criterion to evaluate a VEH. Generally, the VEH design is required to be as compact as possible with the power performance satisfied. The minimized volume not only makes the VEH adapt to the applications with space restrictions but also increases the power density of the device.

- Mechanical quality factor $Q_{m}$.

For a normal linear $\mathrm{VEH}$, the maximum available power is inversely proportional to the mechanical damping coefficient $\mu_{m}$. Therefore, a structure with a high $Q_{m}$ is inclined to have a better power limitation, which can significantly improve the rating of the VEH as indicated in the discussions about PNMA. However, higher $Q_{m}$ will increase the motion space and decrease the available power density. Therefore, a compromise between $Q_{m}$ and $V_{\text {tot }}$ is to be found.

These three aspects reflect the most important properties of a linear VEH. Besides, the bandwidth information is an important complementary aspect. Moreover, it is related to $Q_{m}$ for a linear VEH for which the requirement of large bandwidth is usually in conflict with the demand for maximum power.

\section{A new figure of merit for wideband VEHs}

In practice, the realistic vibration frequency spectrums are broadband and varying from time to time. A lot of examples exhibit frequent variations of the environment vibrations, which bring great challenges to the VEHs. An obvious example is that the acceleration signal acquired at a car wheel is varying 
when driven under different road conditions, urban or rustic [51]. When the natural frequency of the linear oscillator is not inside the favorable range of the excitation, as shown in figure 4 (at time 2), the harvested power is very small. It means that the power of the linear VEHs with a single optimal frequency would fluctuate drastically and affect the normal working of the circuit to be supplied. To have stable and sustainable power output, a generator with wideband response is desired. In contrast to the linear VEH, the wideband VEHs show much stronger adaptabilities with relatively steady harvested power as indicated in figure 4 for both excitation cases at time 1 and time 2 . The foreseeable or stable performance in complex application environments with fluctuating excitations is a critical characteristic, which makes the wideband VEHs more trustable.

In general, the rating of the linear VEHs mainly relies on the maximum usable power at the resonant frequency as seen in the analyses of the figures (PNMA, NPD and $\mathrm{FoM}_{\mathrm{v}}$ ). As a consequence, the attention is focused on the single resonant frequency where the peak power is obtained for linear VEHs. In addition, a compact design is a complementary key issue to get as high power density as possible. The analyses and discussions about some classic figures underline three critical issues for evaluating linear VEHs: $E_{H}, Q_{m}$ and $V_{t o t}$. It provides important information for reference when assessing wideband VEHs. However, more factors are to be accounted for wideband VEHs than for linear ones.

For wideband VEHs, instead of the single optimal working frequency of the linear VEHs, the concern is turned to the performance robustness and stability. In other words, the wideband VEHs' capability of maintaining stable and reliable power output over a variety of working conditions is expected. We focus here on the variations of the operation frequencies over a wide range. Hence, it is more reasonable to evaluate the wideband VEHs by taking the operation bandwidth and the average power in this range as key criteria instead of the maximum power point.

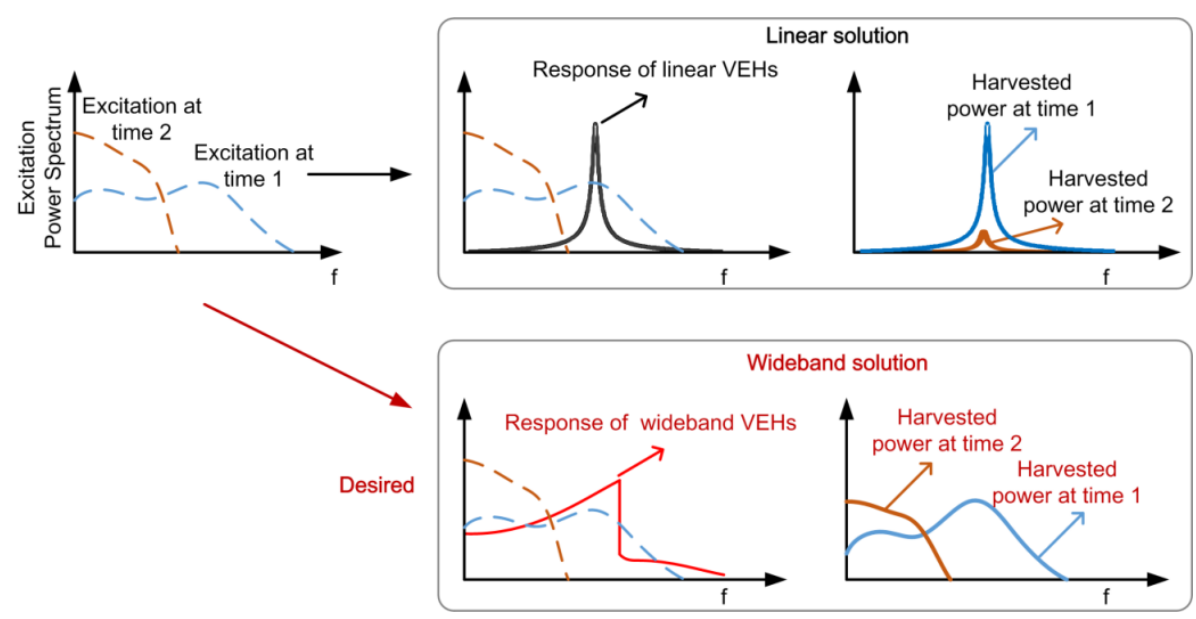

Figure 4. Schematic figure: benefits of wideband generators.

\subsection{New FoM definition}

After overall considerations, a new $\mathrm{SFoM}_{\mathrm{BW}}$ (Systematic Figure of Merit with Bandwidth information) for evaluating wideband VEHs is proposed based on the critical issues explored for linear VEHs with some necessary revisions and special concern on the bandwidth. It is defined as: 
$\operatorname{SFOM}_{B W}=E_{H W} \times \frac{\Delta f}{f_{c}} \times Q_{m} \times \rho_{e f f}$

Here, $E_{H W}$ is the redefined energy harvester effectiveness for wideband VEHs, $\Delta f / f_{c}$ is the bandwidth normalized by the center frequency $f_{c}$ (for the bandwidth definition, $f_{c}=\left(f_{L}+f_{H}\right) / 2, \Delta f=f_{H}-f_{L} . f_{H}$ and $f_{L}$ represent the high end frequency and the low end frequency of the operation band respectively), $Q_{m}$ is the mechanical quality factor and $\rho_{\text {eff }}=M / V_{\text {tot }}$ is the effective mass density accounted for the volume compactness requirement. As mentioned before, $V_{t o t}$ should comprise the motion space and the occupied volume of all the functional parts. To consider the actual volume after installation, it is sometimes more proper to use the minimum block or cylinder volume that can cover the required volume.

$E_{H W}$ derives from the original definition of the energy harvester effectiveness given in equation (18) for linear VEHs. It is presented as follows:

$$
E_{H W}=\frac{P_{a v}}{P_{\lim }}=\frac{P_{a v}}{\frac{M^{2} \gamma_{0}^{2}}{8 \mu_{m}}}=\frac{P_{a v}}{\frac{M \gamma_{0}^{2}}{16 \xi_{m} \omega_{0}}}=\frac{8 P_{a v} \omega_{0}}{M \gamma_{0}^{2} Q_{m}}
$$

A slight adjustment is performed for better appraising of the wideband VEH's performance stability, replacing the usable power output with the average power $P_{a v}$ over the operation band. For the denominator, the maximum available power $P_{\text {lim }}$ of the corresponding linear VEH with the resonant frequency equal to the center frequency $\omega_{0}=1 /\left(2 \pi f_{c}\right)$ is still used as the reference with the same excitation $\gamma_{0}$, mechanical quality factor $Q_{m}=1 /\left(2 \xi_{m}\right)$ and mass $M$. Although a new definition of the energy harvester effectiveness is developed for wideband VEHs, $E_{H W}$ is considered as the key criterion for evaluating the performance of the wideband VEH's electronic part as before, since better $P_{a v}$ is still obtained for the proper configuration of the electromechanical coupling coefficient, the interface circuit and the load to keep the electronic damping at a relatively optimal level.

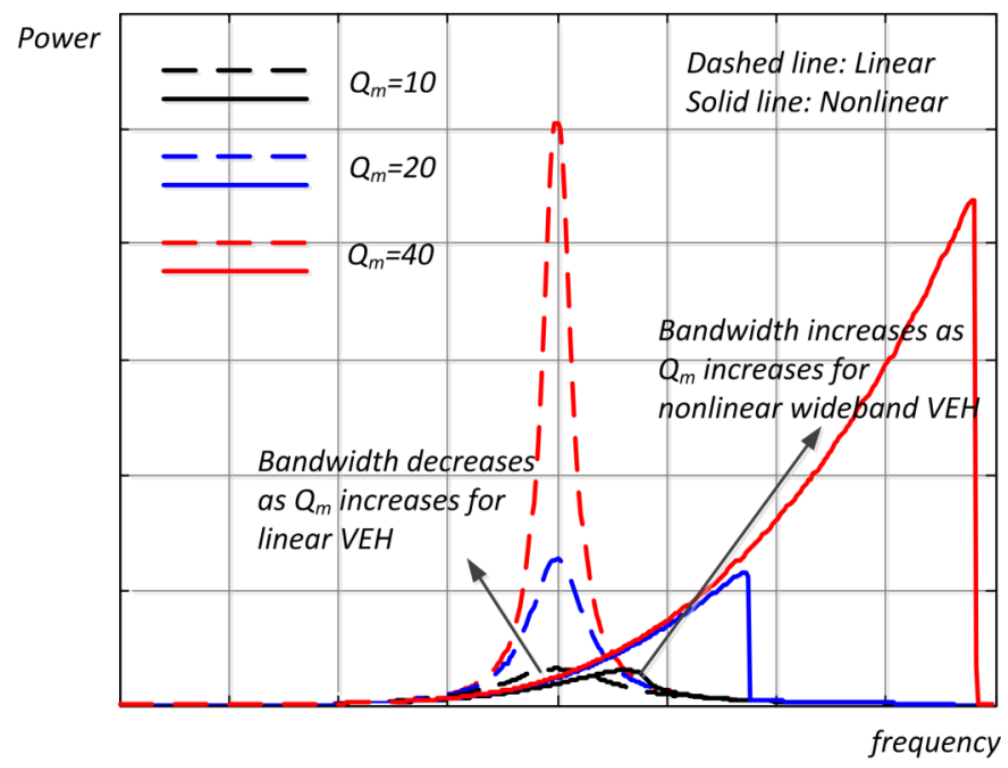

Figure 5. Influence of $Q_{m}$ on a hardening Duffing harvester $\ddot{x}+\omega_{0}{ }^{2} x+2\left(\omega_{0} / Q_{m}\right) \dot{x}+k_{n} x^{3}=\gamma(t)$ and an equivalent linear VEH $\ddot{x}+\omega_{0}{ }^{2} x+2\left(\omega_{0} / Q_{m}\right) \dot{x}=\gamma(t)$ with the electronic damping equal to the mechanical damping. 
$\Delta f / f_{c}$ represents the operation bandwidth, which is one of the key parameters of a wideband VEH. It indicates the important information about the bandwidth widening of the wideband VEHs compared with the linear ones. Various solutions to increase the VEH's bandwidth have been proposed, such as the nonlinear generators, the multimodal generators, the resonance tuning method, the frequency up-conversion method and so on. However, the bandwidth improvement effect is varied with the solutions, the architectures, or the structure parameters etc. Then $\Delta f / f_{c}$ could be used as a good index for the appraisement of the bandwidth widening effect.

It is also worthy of note that high $Q_{m}$ may have a positive influence on the bandwidth for the wideband VEHs. This is different from the linear VEH case in which high $Q_{m}$ favors for the maximum power but hinders the bandwidth. The conflicting effect is clearly demonstrated by the examples in figure 5. The dynamic power responses of several monostable hardening Duffing harvesters with different $Q_{m}$ are presented in comparison with the results of the corresponding linear equivalent harvesters for the same forward sweep excitation. It is shown that higher $Q_{m}$ leads to consistent improvements of the bandwidth and the responses for the Duffing harvesters, but conflicted effects of narrowed bandwidth and increased response for the linear VEHs.

$\mathrm{SFoM}_{\mathrm{BW}}$ provides a systematic evaluation on wideband VEHs as $\mathrm{FoM}_{\mathrm{v}}$ does for linear VEHs. Furthermore, this evaluation is well decoupled into four aspects: $E_{H W}, Q_{m}, \Delta f / f_{c}$ and $\rho_{\text {eff. }}$. The specific contribution ratio of each part on the final value of $\mathrm{SFoM}_{\mathrm{BW}}$ is clearly exhibited, displaying the important information on what kinds of improvement are required. In general, the definition of SFoM $_{\mathrm{BW}}$ implies that a good wideband VEH should possess high harvested power over a wide operation band with a high-quality and compact structure.

Substituting equation (25) into equation (24), $\mathrm{SFoM}_{\mathrm{BW}}$ can be simplified as:

$$
S F_{O M} M_{B W}=\frac{8 P_{a v} \omega_{0}}{M \gamma_{0}^{2} Q_{m}} \times \frac{\Delta f}{f_{c}} \times Q_{m} \times \frac{M}{V_{\text {tot }}}=\frac{16 \pi P_{a v} \Delta f}{\gamma_{0}^{2} V_{\text {tot }}}
$$

which is similar to the expression of NPD. To some extent, $\mathrm{SFoM}_{\mathrm{BW}}$ reflects the wideband VEH's energy density which is the product of the average power density and the bandwidth.

\subsection{Evaluations of $\mathrm{SFoM}_{B W}$ for some wideband VEHs}

Since the nonlinear generators have distinct responses for different excitations, the determination of the bandwidth and the average power is different from the linear generators. It exhibits strong complexities and lacks a common criterion. Different views might exist, depending on the developer's specific choice. However, a general strategy is suggested when using the $\mathrm{SFoM}_{\mathrm{BW}}$ to evaluate the performance of wideband VEHs here. In this section, the idea and the procedure for determining the bandwidth are presented for nonlinear harvester (hardening, softening or bistable). However, they are applicable to other kinds of wideband VEHs as well. Since the responses are related to the applied excitations, the proposed strategy is discussed separately for chirp (sweep), single-frequency and noise excitations.

When subjected to chirp excitations, due to the special hysteresis feature of the nonlinear harvester, the bandwidth needs to be analyzed for the forward and reverse sweep respectively as shown by the power responses of a bistable wideband harvester in figure 6 . The two vertical arrows represent the jump direction for the forward sweep and the reverse sweep respectively. For the forward 
sweep, the harvested power peak $\left(P_{k F}, f_{k F}\right)$ can be found from the power responses. Next, two half peak-power points $\left\{\left(P_{k F} / 2, f_{L F}\right)\right.$ and $\left.\left(P_{k F} / 2, f_{H F}\right), f_{L F}<f_{k F}<=f_{H F}\right\}$ are determined. For the reverse sweep, three corresponding parameters $\left\{\left(P_{k R}, f_{k R}\right),\left(P_{k R} / 2, f_{L R}\right)\right.$ and $\left.\left(P_{k R} / 2, f_{H R}\right), f_{L R}<f_{k R}<=f_{H R}\right\}$ can be found as well. Then the total bandwidth of the wideband VEH is defined as $\Delta f=f_{H F}-f_{L R}$ with the center frequency $f_{c}=\left(f_{H F}+f_{L R}\right) / 2$. This definition is to cover the favorable range for both forward and backward sweep responses. It escapes from the embarrassing situation that the range is not superposed if using the usual $3 \mathrm{~dB}$ method to determine the bandwidth for forward and reverse sweep excitations independently. Although the presented example is for a bistable generator, it is also applicable for the monostable hardening or softening generators. With the bandwidth determined, the average power over the range can be calculated as:

$$
P_{a v}=\frac{1}{\Delta f} \int_{f_{L R}}^{f_{H F}} P_{R}(f) p_{1}(f)+P_{F}(f) p_{2}(f) d f
$$

in which $P_{R}(f)$ and $P_{F}(f)$ are the reverse and forward power response respectively. It is worth mentioning that if the way to calculate $\Delta f$ shown in figure 6 tends to increase or overestimate the bandwidth, it is compensated by the fact that it also decreases the average power. Considering that the forward and reverse responses represent two possible stable motion solutions of the harvester, the final status is determined by the initial condition. Then the probability for each response can be determined from the basin of attraction at the specific frequency with the excitation fixed and the probability distribution of the initial conditions. In equation $(27), p_{l}(f)$ denotes the probability to obtain a reverse sweep response and $p_{2}(f)$ is the probability for a forward sweep response.

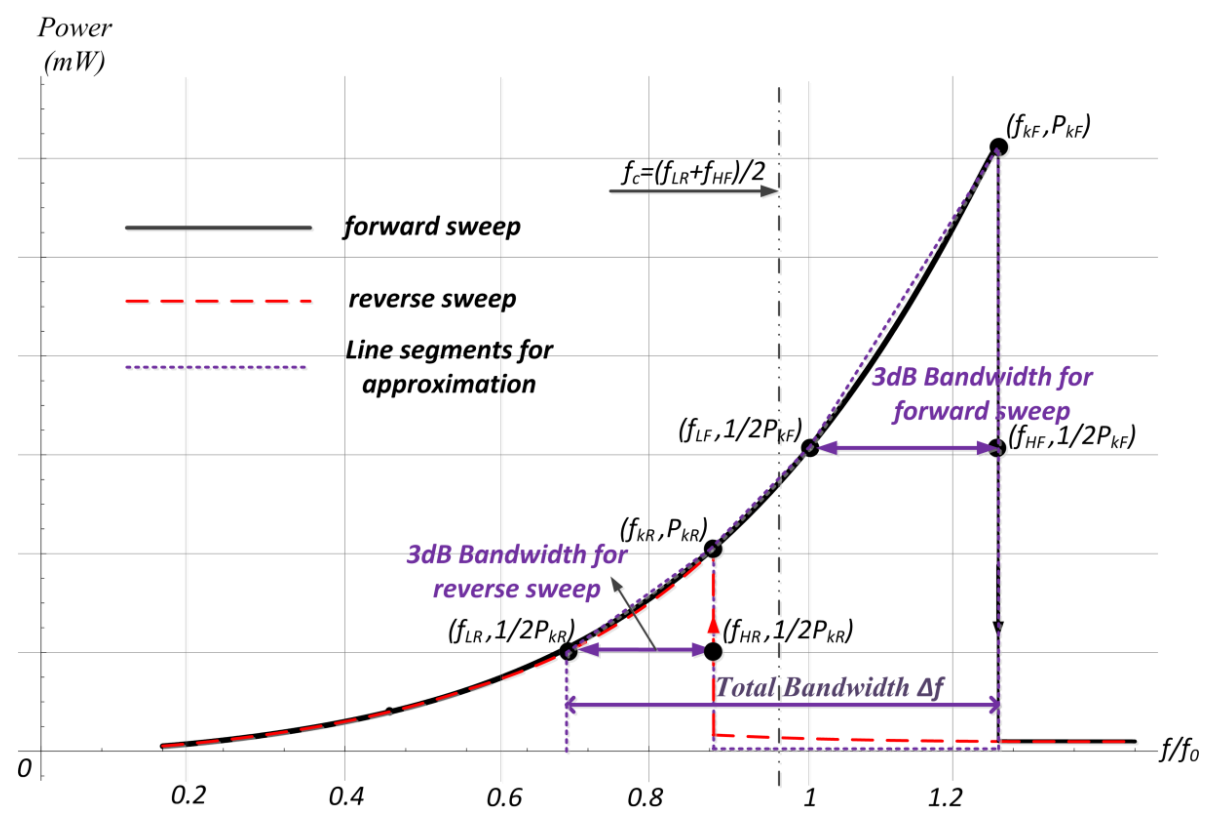

Figure 6. Bandwidth definition and average power approximation for a bistable generator.

For simplifications, it is assumed that $p_{1}(f)=p_{2}(f)=1 / 2$ here. As a further step, the power curve is approximated with some linear segments (purple dashed lines) as shown in figure 6 . If the power is less than $\min \left(P_{k F}, P_{k R}\right) / 2$, it is considered as zero with the approximation of horizontal lines. Then the average power is estimated as: 


$$
P_{a v}=\frac{1}{2 \Delta f}\left(\frac{3}{2} P_{k R}\left(f_{H R}-f_{L R}\right)+\frac{1}{2}\left(\frac{1}{2} P_{k F}+P_{k R}\right)\left(f_{L F}-f_{K R}\right)+\frac{3}{4} P_{k F}\left(f_{H F}-f_{L F}\right)\right)
$$

for hardening and bistable generators or

$$
P_{a v}=\frac{1}{2 \Delta f}\left(\frac{3}{2} P_{k F}\left(f_{H F}-f_{L F}\right)+\frac{1}{2}\left(\frac{1}{2} P_{k R}+P_{k F}\right)\left(f_{L F}-f_{H R}\right)+\frac{3}{4} P_{k R}\left(f_{H R}-f_{L R}\right)\right)
$$

for softening generators.

However, it is worthy of note that this approximation is only effective when the error induced by the linear approximation can be neglected. When the power curve is not suitable for the linear approximation, other approximation method needs to be used.

If the power response is obtained with the single-frequency harmonic excitation whose frequency is changed step by step with the harvester starting from the zero initial condition, the bandwidth is proposed to be defined by the points $\left\{\left(P_{k}, f_{k}\right),\left(P_{k} / 2, f_{L}\right)\right.$ and $\left.\left(P_{k} / 2, f_{H}\right), f_{L}<f_{k}<=f_{H}\right\}$ with $P_{k}$ representing the peak power and $f_{L}, f_{k}, f_{H}$ representing the corresponding frequency positions. Then the average power is calculated as:

$$
P_{a v}=\frac{1}{\Delta f} \int_{f_{L}}^{f_{H}} P(f) d f
$$

A similar strategy can also be used for bandlimited noise excitations.

Table 1 lists some literature generators with the calculated $\mathrm{SFoM}_{\mathrm{BW}}$ figure. Some parameters of the generators in the literature are estimated from the provided response curves so that inaccuracies may exist. It however confirms that the proposed figure of merit offers a tool for the evaluation of the performance of nonlinear generators. Moreover, PNMA, NPD and $\mathrm{FoM}_{\mathrm{v}}$ are also calculated for comparison. It is interesting to find that NPD and $\mathrm{FoM}_{\mathrm{v}}$ present relatively similar results if sorting the VEHs by the corresponding values. It is because the two figures contain all the essential elements for linear VEHs. PNMA excludes the volume information thus deviates from the trends indicated by NPD or $\mathrm{FoM}_{\mathrm{v}}$ for some VEHs. However, the lack of information on the bandwidth and on the average

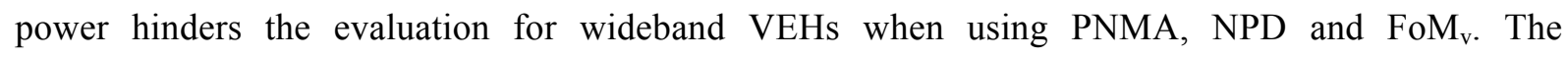
appraisement results of $\mathrm{SFoM}_{\mathrm{BW}}$ are different from the linear VEH figures and believed to be more rational. 


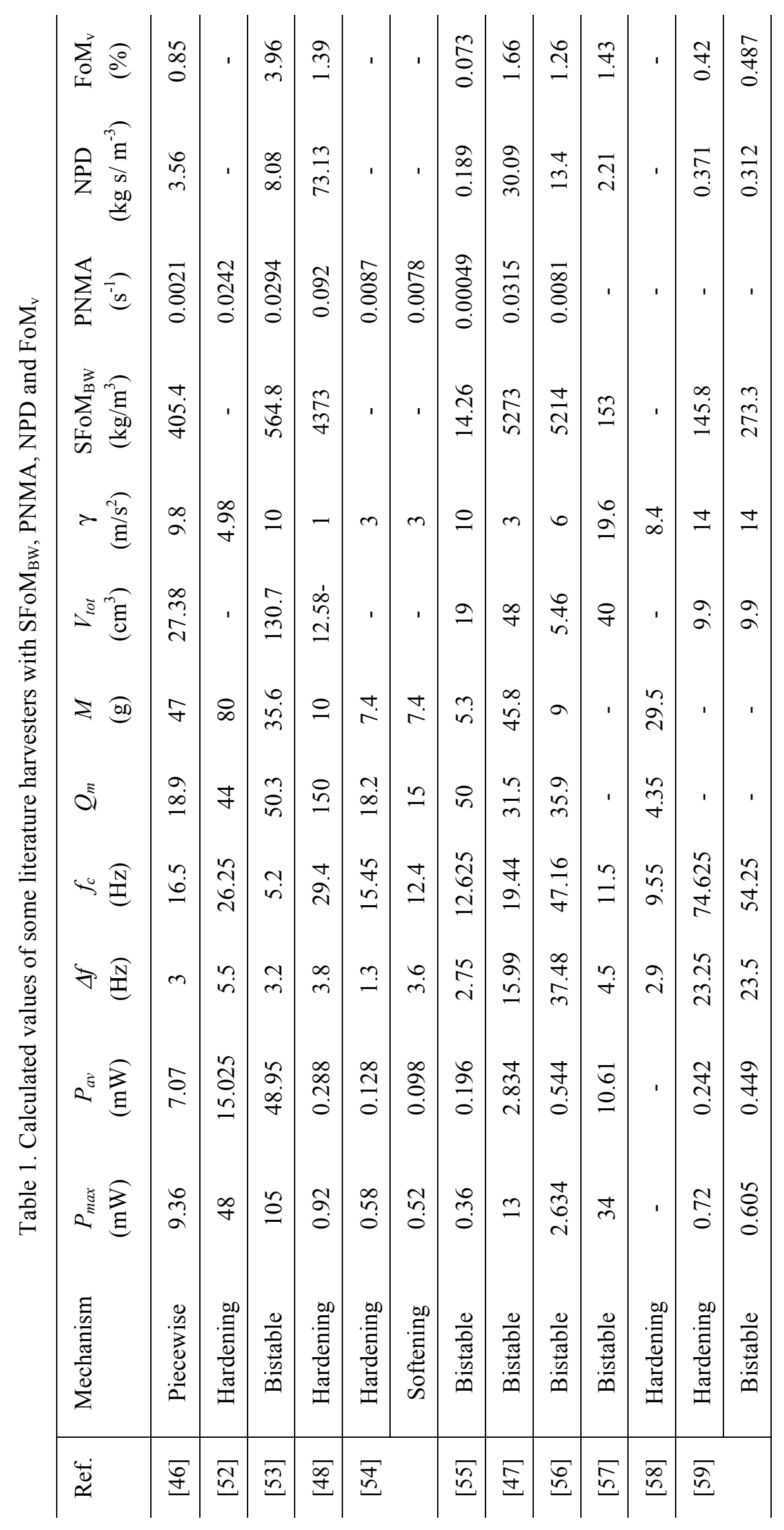




\section{Conclusion}

In this article, a new systematic figure of merit named $\mathrm{SFoM}_{\mathrm{BW}}$ oriented towards wideband VEHs is developed from the detailed study of the classic figures for linear VEHs. This study demonstrates that a good evaluation figure is required to appraise three aspects $\left(E_{H}, Q_{m}, V_{t o t}\right)$ in a proper way.

Generally, good assessments with classic figures such as PNMA, NPD and $\mathrm{FoM}_{\mathrm{v}}$ are in favor of linear VEHs exhibiting optimal energy extraction ability $\left(E_{H}=1\right)$, optimal configuration of the mechanical damping, and optimal use of the device volume. However, the evaluation of linear figures focuses on the peak performance at a single frequency, usually the resonant frequency. It discords with the performance target of wideband VEHs, which is to increase the operation band and the average power in this range. Special consideration has to be given to these two aspects.

Devoted to the feature and the performance target of wideband VEHs, SFoM $\mathrm{BW}_{\mathrm{BW}}$ integrates the essential factors for evaluation including the amended energy harvester effectiveness $E_{H W}$, the normalized bandwidth $\Delta f / f_{c}$, the mechanical quality factor $Q_{m}$ and the effective density $\rho_{\text {eff. The }}$ influence of each aspect is analyzed and discussed. The utilization is then explicated and demonstrated for some examples and compared with some former figures for some wideband VEHs from the literature. It shows that $\mathrm{SFoM}_{\mathrm{BW}}$ provides different evaluation results from those figures with more overall consideration for wideband VEHs. Unfortunately, a lot of wideband VEHs published results didn't provide enough information to calculate the $\mathrm{SFoM}_{\mathrm{BW}}$ value, especially for the power response curve and the volume. Detailed and completed information about the essential factors to evaluate the wideband VEHs should be provided when performing the investigations. Moreover, it is worth mentioning that the performance of the wideband VEHs, including the average power and the bandwidth, will vary with the excitation amplitude. Thus, the SFoMBW value only accounts for a selected excitation pattern. If the values are obtained for different excitations, a better understanding of the wideband VEHs could be found. However, it requires painstaking work and it can be unachievable. Though, it is still meaningful that SFoMBW provides a good evaluation tool for the wideband VEHs and points out the direction of the improvement about the concerned wideband VEHs.

\section{Acknowledgements}

This work has been supported by the French National Research Agency (ANR) under grant No. ANR-11-JS09-002-01 (REVilaBa Project), the National Science Foundation of China (No.51505395) and the Fundamental Research Funds for the Central Universities of China (No. A0920502051514-5).

\section{References}

[1] Zhang Y, Zhang F, Shakhsheer Y, et al. 2013 A Batteryless $19 \mu \mathrm{W}$ MICS/ISM-Band Energy Harvesting Body Sensor Node SoC for ExG Applications IEEE Journal of solid-state circuits 48(1) 199-213.

[2] Dunn-Rankin D, Leal E M, and Walther D C. 2005 Personal power systems Progress in Energy and Combustion Science 31(5-6) 422-465.

[3] Jang S, Jo H, Cho S, Mechitov K, et al. 2010 Structural health monitoring of a cable-stayed bridge using smart sensor technology: deployment and evaluation Smart Structures and systems 6 439-459.

[4] Ruiz-Garcia L, Lunadei L, Barreiro P and J I Robla. 2009 A review of wireless sensor technologies and applications in agriculture and food industry: state of the art and current trends Sensors (Basel, Switzerland), 9(6) $4728-50$.

[5] Oliveira L M and Rodrigues J J. 2011 Wireless Sensor Networks: A Survey on Environmental Monitoring Journal of Communications, 6(2) 143-151. 
[6] Ko J G, Lu C, and Srivastava M B. 2010 Wireless sensor networks for healthcare Proceedings of the IEEE, 98(11) 1947-1960

[7] Hande A, Polk T, Walker W and Bhatia D 2007 Indoor solar energy harvesting for sensor network router nodes Microprocessors and Microsystems 31 420-432.

[8] Ujihara M, Carman G P and Lee D G 2007 Thermal energy harvesting device using ferromagnetic materials Applied Physics Letters 91(9) 093508.

[9] Roundy S, Wright P K and Rabaey J 2003 A study of low level vibrations as a power source for wireless sensor nodes Computer Communications 26 1131-1144

[10] Beeby S P, Tudor M J and White N M 2006 Energy harvesting vibration sources for microsystems applications Measurement Science and Technology 17(12) R175-R195.

[11] Anton S R and Sodano H A 2007 A review of power harvesting using piezoelectric materials (2003-2006) Smart Materials and Structures 16(3) R1-R21.

[12] Liu W Q 2014 Conception d'un dispositif de recuperation d'energie vibratoire large bande PhD thesis, Université de Savoie.

[13] Xu JW, Shao WW, Kong FR, et al. 2010 Right-angle piezoelectric cantilever with improved energy harvesting efficiency Applied Physics Letters 96(15) 152904.

[14] Arroyo E, Badel A and Formosa F 2013 Energy harvesting from ambient vibrations: Electromagnetic device and synchronous extraction circuit Journal of Intelligent Material Systems and Structures 24(16) 2023-2035.

[15] Guan QC, Ju B, Xu JW, et al. 2013 Improved strain distribution of cantilever piezoelectric energy harvesting devices using H-shaped proof masses Journal of Intelligent Material Systems and Structures 24(9) 1059-1066.

[16] Arroyo E, Badel A, Formosa F, Wu Y and Qiu J 2012 Comparison of electromagnetic and piezoelectric vibration energy harvesters: model and experiments Sensors and Actuators A: Physical 183 148-156.

[17] Badel A, Guyomar D, Lefeuvre E and Richard C 2005 Efficiency enhancement of a piezoelectric energy harvesting device in pulsed operation by synchronous charge inversion Journal of Intelligent Materials Systems and Structures 16 889-901.

[18] Liang J R and Liao W H 2011 On the Influence of Transducer Internal Loss in Piezoelectric Energy Harvesting with SSHI Interface Journal of Intelligent Material Systems and Structures 22(5) 503-512.

[19] Lien I C, Shu Y C, Wu W J, Shiu S M and Lin H C 2010 Revisit of series-SSHI with comparisons to other interfacing circuits in piezoelectric energy harvesting Smart Materials and Structures 19(12) 125009.

[20] Lefeuvre E, Badel A, Richard C and Guyomar D 2005 Piezoelectric energy harvesting device optimization by synchronous electric charge extraction Journal of Intelligent Materials Systems and Structures 16 865-76.

[21] Lallart M, Garbuio L, Petit L, Richard C and Guyomar D J 2008 Double synchronized switch harvesting (DSSH): a new energy harvesting scheme for efficient energy extraction IEEE transactions on ultrasonics, ferroelectrics, and frequency control 55(10) 2119-2130.

[22] Wu Y, Badel A, Formosa F, Liu W and Agbossou A E 2013 Piezoelectric vibration energy harvesting by optimized synchronous electric charge extraction Journal of Intelligent Materials Systems and Structures 24(12) 1445-58.

[23] Liu W Q, Badel A, Formosa F, Wu Y and Agbossou A. 2013 Wideband energy harvesting using a combination of an optimized synchronous electric charge extraction circuit and a bistable harvester Smart Materials and Structures 22(12) 125038.

[24] Arroyo E and Badel A. 2011 Electromagnetic vibration energy harvesting device optimization by synchronous energy extraction Sensors and Actuators A: Physical 171(2) 266-273.

[25] Cao X and Chiang W J. 2007 Electromagnetic energy harvesting circuit with feedforward and feedback 
DC-DC PWM boost converter for vibration power generator system IEEE Transactions on Power Electronics 22(2) 679-685.

[26] Roundy S 2005 On the Effectiveness of Vibration-based Energy Harvesting Journal of Intelligent Material Systems and Structures 16(10) 809-823. doi:10.1177/1045389X05054042

[27] Mitcheson P and Green T. 2004 Architectures for vibration-driven micropower generators Journal of Microelectromechanical Systems 13(3) 429-440.

[28] Mitcheson P, Yeatman E M, Rao G K and Green T C. 2008 Human and Machine Motion for Wireless Electronic Devices Proceedings of the IEEE 96(9) 1457-1486.

[29] Beeby S P, Torah R N, Tudor M J, Glynne-Jones P et al. 2007 A micro electromagnetic generator for vibration energy harvesting Journal of Micromechanics and Microengineering 17(7) 1257-1265.

[30] Tang L, Yang Y and CK Soh. 2010 Toward broadband vibration-based energy harvesting Journal of Intelligent Material Systems and structures 21(18) 1867.

[31] Twiefel J and Westermann H. 2013 Survey on broadband techniques for vibration energy harvesting Journal of Intelligent Material Systems and Structures 24(11) 1291-1302.

[32] Pellegrini S P, Tolou N, Schenk M and Herder J L 2012 Bistable vibration energy harvesters: A review Journal of Intelligent Material Systems and Structures DOI: 10.1177/1045389X12444940.

[33] Zhu D, Tudor M J and Beeby S P 2010 Strategies for increasing the operating frequency range of vibration energy harvesters: a review Measurement Science and Technology 21(2) 022001.

[34] Leland E S and Wright P K. 2006 Resonance tuning of piezoelectric vibration energy scavenging generators using compressive axial preload Smart Materials and Structures 15 1413-1420.

[35] Challa V R, Prasad M G and Fisher F T. 2011 Towards an autonomous self-tuning vibration energy harvesting device for wireless sensor network applications Smart Materials and Structures 20(2) 025004.

[36] Pillatsch P, Yeatman E M, and Holmes A S. 2012 A scalable piezoelectric impulse excited energy harvester for human body excitation Smart Materials and Structures 21(11) 115018.

[37] Galchev T, Kim H and K Najafi. 2011 Micro power generator for harvesting low-frequency and nonperiodic vibrations Journal of Microelectromechanical Systems 20(4) 852-866.

[38] Ferrari M, Ferrari V, Guizzetti M, Marioli D and Taroni A. 2008 Piezoelectric multifrequency energy converter for power harvesting in autonomous microsystems Sensors and Actuators A: Physical 142329 335.

[39] Tadesse Y and Priya S. 2008 Multimodal Energy Harvesting System: Piezoelectric and Electromagnetic Journal of Intelligent Material Systems and Structures 20(5) 625-632.

[40] Harne R L and Wang K W 2013 A review of the recent research on vibration energy harvesting via bistable systems Smart Materials and Structures 22(2) 023001.

[41] Cottone F, Vocca H and Gammaitoni L 2009 Nonlinear Energy Harvesting Physical Review Letters 102(8) 080601.

[42] Ferrari M, Ferrari V, Guizzetti M, Andò B, Baglio S and Trigona C 2010 Improved energy harvesting from wideband vibrations by nonlinear piezoelectric converters Sensors and Actuators A: Physical 162(2) 425-31.

[43] Liu W Q, Badel A, Formosa F, Wu Y and Agbossou A. 2014 Self-powered nonlinear harvesting circuit with a mechanical switch structure for a bistable generator with stoppers Sensors and Actuators A: Physical 216 106-115.

[44] Liu H, Lee C, Kobayashi T, Tay C J and Quan C. 2012 Investigation of a MEMS piezoelectric energy harvester system with a frequency-widened-bandwidth mechanism introduced by mechanical stoppers Smart Materials and Structures 21(3) 035005

[45] Erturk A and Inman D J 2011 Broadband piezoelectric power generation on high-energy orbits of the bistable 
Duffing oscillator with electromechanical coupling Journal of Sound and Vibration 330(10) 2339-53.

[46] Ashraf K, Khir M H Md, Dennis J O and Baharudin Z. 2013 A wideband, frequency up-converting bounded vibration energy harvester for a low-frequency environment Smart Mater. Struct. 22(2) 025018.

[47] Liu W Q, Badel A, Formosa F, Wu Y and Agbossou A 2013 Novel piezoelectric bistable oscillator architecture for wideband vibration energy harvesting Smart Materials and Structures 22(3), 035013.

[48] Sebald G, Kuwano H, Guyomar D and Ducharne B 2011 Simulation of a Duffing oscillator for broadband piezoelectric energy harvesting Smart Materials and Structures 20075022.

[49] Stanton S C, Owens B A M and Mann B P. 2012 Harmonic balance analysis of the bistable piezoelectric inertial generator Journal of Sound and Vibration 331(15) 3317-3327.

[50] Lefeuvre E, Badel A, Richard C, Petit L and Guyomar D 2006 A comparison between several vibration-powered piezoelectric generators for standalone systems Sensors and Actuators A: Physical 126(2) 405-16.

[51] Neri I, Travasso F, Mincigrucci R, et al. 2012 A real vibration database for kinetic energy harvesting application Journal of Intelligent Material Systems and Structures 23(18) 2095-2101.

[52] Barton D, Burrow S and Clare L. 2010 Energy Harvesting From Vibrations With a Nonlinear Oscillator. Journal of Vibration and Acoustics, 132(2) 021009

[53] Mann B P and Owens B A 2010 Investigations of a nonlinear energy harvester with a bistable potential well Journal of Sound and Vibration 329(9) 1215-26.

[54] Stanton S C, McGehee C C and Mann B P. 2009 Reversible hysteresis for broadband magnetopiezoelastic energy harvesting Applied Physics Letters 95(17) 174103.

[55] Stanton S C, Mcgehee C C and Mann B P 2010 Nonlinear dynamics for broadband energy harvesting: Investigation of a bistable piezoelectric inertial generator Physica D 239(10) 640-53.

[56] Liu W Q, Badel A, Formosa F, Wu Y, N Bencheikh and Agbossou A. 2015 A wideband integrated piezoelectric bistable generator: Experimental performance evaluation and potential for real environmental vibrations Journal of Intelligent Material Systems and Structures 26(7) 872-877.

[57] Arrieta A and Hagedorn P 2010 A piezoelectric bistable plate for nonlinear broadband energy harvesting Applied Physics letter 97104102.

[58] Mann B P and Sims N D 2009 Energy harvesting from the nonlinear oscillations of magnetic levitation Journal of Sound and Vibration 319 515-530.

[59] Masana R and Daqaq M F 2011 Relative performance of a vibratory energy harvester in mono- and bistable potentials Journal of Sound and Vibration 330(24) 6036-52. 